KYUNGPOOK Math. J. 55(2015), 871-883

http://dx.doi.org/10.5666/KMJ.2015.55.4.871

pISSN 1225-6951 eISSN 0454-8124

(C) Kyungpook Mathematical Journal

\title{
On Divisorial Submodules
}

\author{
Ahmad Yousefian Darani* and Mahdi Rahmatinia \\ Department of Mathematics and Applications, University of Mohaghegh Ardabili, \\ P. O. Box 179, Ardabil, Iran \\ e-mail : yousefian@uma.ac.ir and m.rahmati@uma.ac.ir
}

Abstract. This paper is devoted to study the divisorial submodules. We get some equivalent conditions for a submodule to be a divisorial submodule. Also we get equivalent conditions for $(N \cap L)^{-1}$ to be a ring, where $N, L$ are submodules of a module $M$.

\section{Introduction}

Throughout this paper all rings are considered commutative rings with identiry and all modules are considered unitary. Let $R$ be a commutative ring with identity and let $M$ be an $R$-module. $M$ is called a multiplication module if every submodule $N$ of $M$ has the form $I M$ for some ideal $I$ of $R$. Let $M$ be a multiplication $R$ module and $N$ a submodule of $M$. Then $N=I M$ for some ideal $I$ of $R$. Hence $I \subseteq\left(N:_{R} M\right)$ and so $N=I M \subseteq\left(N:_{R} M\right) M \subseteq N$. Therefore $N=\left(N:_{R} M\right) M$ [8]. Let $R$ be an integral domain, $M$ a faithful multiplication $R$-module and $N, L$ submodules of $M$. Then $\left(N:_{R} M\right)\left(L:_{R} M\right)=\left(\begin{array}{ll}N L:_{R} & M\end{array}\right)$ [7, Lemma 3.6]. Therefore $N L=\left(N L:_{R} M\right) M$. Thus we get $N^{n}=\left(N^{n}:_{R} M\right) M$. An $R$-module $M$ is called a cancellation module if $I M=J M$ for two ideals $I$ and $J$ of $R$ implies $I=J$ [3]. By [17, Corollary 1 to Theorem 9], finitely generated faithful multiplication modules are cancellation modules. It follows that if $M$ is a finitely generated faithful multiplication $R$-module, then $\left(I N:_{R} M\right)=I\left(N:_{R} M\right)$ for all ideals $I$ of $R$ and all submodules $N$ of $M$. If $R$ is an integral domain and $M$ a faithful multiplication $R$-module, then $M$ is a finitely generated $R$-module [9].

Let $S$ be the set of all non-zero divisors of $R$ and $T(R)=R_{S}$ the total quotient ring of $R$. For a nonzero ideal $I$ of $R$, let $I^{-1}=\{x \in T(R): x I \subseteq R\}$. An ideal $I$

* Corresponding Author.

Received August 22, 2014; accepted May 13, 2015

2010 Mathematics Subject Classification: 16N99, 16 S99.

Key words and phrases: divisorial submodule, prime submodule, radical submodule, multiplication module.

This work was supported by the University of Mohaghegh Ardabili. 
of $R$ is called invertible, if $I I^{-1}=R$. Let $M$ be an $R$-module and set

$$
T=\{t \in S: \text { for all } m \in M, t m=0 \text { implies } m=0\} .
$$

Then $T$ is a multiplicatively closed subset of $R$ with $T \subseteq S$, and if $M$ is torsionfree then $T=S$. In particular, $T=S$ if $M$ is a faithful multiplication $R$-module [9, Lemma 4.1]. Let $N$ be a nonzero submodule of $M$. Then we write $N^{-1}=$ $\left(M:_{R_{T}} N\right)=\left\{x \in R_{T}: x N \subseteq M\right\}$ and $N_{\nu}=\left(N^{-1}\right)^{-1}$. Then $N^{-1}$ is an $R$ submodule of $R_{T}, R \subseteq N^{-1}$ and $N N^{-1} \subseteq M$. We say that $N$ is invertible in $M$ if $N N^{-1}=M$. Clearly $0 \neq M$ is invertible in $M$. If $I$ is an invertible ideal of $R$ then $I M$ is invertible in $M$ and the converse is true if $M$ is a finitely generated faithful multiplication $R$-module [15]. Every invertible submodule $N$ of a finitely generated faithful multiplication $R$-module $M$ is finitely generated faithful multiplication and the converse is true if $R$ is an integral domain [1]. An $R$-module $M$ is called a Dedekind module (resp., Prüfer module) if every nonzero submodule (resp., every nonzero finitely generated submodule) of $M$ is invertible [15]. An $R$-module $M$ is called a valuation module if for all $m, n \in M$, either $R m \subseteq R n$ or $R n \subseteq R m$. Equivalently, $M$ is a valuation module if for all submodules $N$ and $K$ of $M$, either $N \subseteq K$ or $K \subseteq N[4]$.

Following [3], a submodule $N$ of $M$ is called a divisorial submodule of $M$ in case $N=N_{\nu} M$. If $M$ is a finitely generated faithful multiplication $R$-module, then $N_{\nu}=\left(N:_{R} M\right)$. Consequently, $M_{\nu}=R$. Let $M$ be a finitely generated faithful multiplication $R$-module, $N$ a submodule of $M$ and $I$ an ideal of $R$. Then $N$ is a divisorial submodule of $M$ if and only if $\left(N:_{R} M\right)$ is a divisorial ideal of $R$. Also $I$ is divisorial ideal of $R$ if and only if $I M$ is a divisorial submodule of $M$ [2]. If $N$ is an invertible submodule of a faithful multiplication module $M$ over an integral domain $R$, then $\left(N:_{R} M\right)$ is invertible hence a divisorial ideal of $R$. So $N$ is a divisorial submodule of $M$ [2]. If $R$ is an integral domain, $M$ a faithful multiplication $R$-module and $N$ a nonzero submodule of $M$, then $N_{\nu}=\left(N:_{R} M\right)_{\nu}$ [2, Lemma 1]. A submodule $N$ of an $R$-module $M$ is called an idempotent submodule of $M$ if $N=\left(N:_{R} M\right) N$. It is shown that, if $M$ is a multiplication $R$-module and $N$ a submodule of $M$ such that $\left(N:_{R} M\right)$ is an idempotent ideal of $R$, then $N$ is an idempotent submodule of $M$. The converse is true if $M$ is a finitely generated faithful multiplication $R$-module [5, Theorem 3]. We say that a submodule $N$ of $M$ is a radical submodule of $M$ if $N=\sqrt{N}$, where $\sqrt{N}=\sqrt{\left(N:_{R} M\right)} M$. If $a \in \sqrt{N}=\sqrt{\left(N:_{R} M\right)} M$, then $a^{n} \in\left(N:_{R} M\right) M=N$. Also, $N=\left(N:_{R} M\right) M \subseteq$ $\sqrt{\left(N:_{R} M\right)} M=\sqrt{N}$. It is shown that if $R$ is an integral domain, $M$ a faithful multiplication $R$-module and $N$ a submodule of $M$, then $N$ is a radical submodule of $M$ if and only if $\left(N:_{R} M\right)$ is a radical ideal of $R$ [6, Lemma 6]. Let $R$ be a ring and $M$ a finitely generated multiplication $R$-module. Then $Q$ is a $P$-primary submodule of $M$ if and only if $\left(Q:_{R} M\right)$ is a $\left(P:_{R} M\right)$-primary ideal of $R[2$, Lemma 4].

Let $R$ be a an integral domain, $M$ a faithful multiplication $R$-module and $N$ a submodule of $M$. Then $T(N)=\bigcup_{n=1}^{\infty}\left(M:_{K}(N ; M)^{n} M\right)$. Furthermore, if $M$ is 
finitely generated, then $T(N)=\bigcup_{n=1}^{\infty}\left(R:_{K}\left(N:_{R} M\right)^{n}\right)=T\left(\left(N:_{R} M\right)\right)$, [2]. An integral domain $R$ is said to satisfy the trace property $(T P)$ provided that $\operatorname{Tr}(M)=\sum_{f \in \operatorname{Hom}(M, R)} f(M)$ either equals $R$ or is a prime ideal of $R$ for each $R$-module $M$ [11]. An $R$-module $M$ satisfies trace property $(T P)$ if $\operatorname{Tr}_{M}(N)=M$ or $\operatorname{Tr}_{M}(N)$ is a prime submodule for each nonzero submodule $N$ of $M$, where $\operatorname{Tr}_{M}(N)=\sum_{f \in H o m(N, M)} f(N)$, [2]. It is proved that if $R$ is a ring, $M$ an $R$-module and $N$ a submodule of $M$, then $\operatorname{Tr}_{M}(N)^{-1}=\left(\operatorname{Tr}_{M}(N):_{R_{T}} \operatorname{Tr}_{M}(N)\right)$. Moreover if $M$ is a finitely generated faithful multiplication $R$-module, then $\operatorname{Tr}_{M}(N)=N N^{-1}$, [2, Theorem 15].

In section 2, we generalize some properties of dividorial ideals of an integral domain to modules. In Lemma 2.1, we get equivalent conditions for a submodule to be a divisorial submodule. Let $R$ be an integral domain, $M$ a Prüfer faithful multiplication $R$-module and $P$ a maximal submodule of $M$. Then each power of $P$ is a divisorial submodule of $M$ if and only if $P^{2}$ is a divisorial submodule of $M$ (Lemma 2.4). In Theorem 2.5, we imply other equivalent conditions for each power of $P$ to be divisorial submodule. An $R$-module $M$ satisfies radical trace property $(R T P)$ provided $\operatorname{Tr}_{M}(N)=M$ or $\operatorname{Tr}_{M}(N)=\sqrt{N N^{-1}}$ for each submodule $N$ of $M$. In Theorem 2.8, we show that if $R$ is an integral domain and $M$ a faithful multiplication $R$-module, then $M$ satisfies $R T P$ if and only if $R$ satisfies $R T P$. In Theorem 2.9 , we give equivalent conditions for $M$ to satisfies $R T P$. In proposition 2.14, we show that, if $R$ is an integral domain and $M$ a faithful multiplication $R$-module, then $M$ is a discrete Valuation module if and only if each $P$-primary submodule of $M$ is a power of $P$. Let $R$ ba an integral domain and $M$ a faithful multiplication $R$-module. Let $N$ and $K$ be two submodules of $M$ such that $N$ is invertible and $K \subseteq N$. Then there exists an ideal $I$ of $R$ such that $K=I N$ (Lemma 2.15). Let $R$ be an integral domain, $K$ a quotient field of $R$ such that $R \neq K$ and $M$ a Valuation faithful multiplication $R$-module. Then the product of two $P$-primary submodules of $M$ is a $P$-primary submodule of $M$ (Lemma 2.17). In Theorem 2.18, we prove that, if $R$ is an integral domain, $K$ a quotient field of $R$ such that $R \neq K$ and $M$ a Valuation faithful multiplication $R$-module and if $P \neq P^{2}$ is a prime submodule of $M$, then each $P$-primary submodule of $M$ is a power of $P$.

In section 3, we give equivalent conditions for $N^{-1}$ and $L^{-1}$ to be rings, where $N, L$ are submodules of $R$-module $M$. let $R$ be a ring and $N, L$ be two submodules of an $R$-module $M$. Then $N$ and $L$ are coprime if $N+L=M$ [2]. Let $R$ be an integral domain, $M$ a faithful multiplication $R$-module and $N, L$ coprime submodules of $M$. We get equivalent conditions for $(N \cap L)^{-1}$ to be a ring (Theorem 3.1 and Theorem 3.6). In Proposition 3.7, we show that if $R$ is an integral domain, $M$ a faithful multiplication $R$-module and $N$ a radical submodule of $M$ such that $N=K \cap L$ for submodules $K, L$ of $M$, then $N^{-1}$ is a ring if and only if there are radical submodules $K_{1} \supseteq K$ and $L_{1} \supseteq L$ of $M$ such that $N=K_{1} \cap L_{1}$ and $K_{1}^{-1}$ and $L_{1}^{-1}$ are rings. 


\section{Divisorial Submodules}

Compare the next results with [11, Lemma 4.1.8, Lemma 4.1.16, Proposition 4.1.17, Corollary 4.1.18, Theorem 4.1.19, Theorem 4.2.13, Lemma 4.2.14, Lemma 4.2.15 and Lemma 5.3.1]. We start with the following lemma.

Lemma 2.1. Let $R$ be an integral domain and $M$ a Prüfer faithful multiplication $R$-module. If $P$ is a maximal submodule of $M$, then the following are equivalent:

(1) $P$ is a divisorial submodule of $M$;

(2) $P^{n}$ is a divisorial submodule of $M$ for each positive integer $n$;

(3) $P$ is a finitely generated submodule of $M($ i.e. $P$ is an invertible submodule of $M)$.

Proof. (1) $\Rightarrow$ (3) If $P$ is not an invertible submodule of $M$, then $P^{-1}=R,[2$, Proposition 13]. Since $P$ is a divisorial submodule of $M$, then

$$
P=\left(P^{-1}\right)^{-1}=R^{-1}=R
$$

which is a contradiction, because $R$ is invertible. Therefore $P$ is an invertible submodule of $M$.

$(3) \Rightarrow(2)$ If $P$ is a finitely generated submodule of $M$, then so is $P^{n}$ for each positive integer $n$. So $P^{n}$ is an invertible submodule of $M$. Thus $P^{-n}$ is not a subring of $T(R)$, [2, Proposition 11]. Therefore $P^{n}$ is a divisorial submodule of $M$ for each positive integer $n,[2$, Proposition 14].

$(2) \Rightarrow(1)$ It is clear.

Lemma 2.2. Let $R$ be an integral domain, $M$ a faithful multiplication $R$-module and $N$ a submodule of $M$. Then $\left(M:_{R_{T}} T(N)\right)=\bigcap_{n=1}^{\infty}\left(N^{n}\right)_{\nu}$.

Proof. By [2, Lemma 1], we have $\left(N:_{R} M\right)_{\nu}=N_{\nu}$. Therefore

$$
\begin{aligned}
\left(M:_{R_{T}} T(N)\right) & =\left(M:_{R_{T}} \bigcup_{n=1}^{\infty}\left(M:_{R_{T}}\left(N:_{R} M\right)^{n} M\right)\right) \\
& =\left(M:_{R_{T}} \bigcup_{n=1}^{\infty}\left(N:_{R} M\right)^{-n} M\right) \\
& =\bigcap_{n=1}^{\infty}\left(M:_{R_{T}}\left(N:_{R} M\right)^{-n} M\right) \\
& =\bigcap_{n=1}^{\infty}\left(R:_{T(R)}\left(N:_{R} M\right)^{-n}\right)=\bigcap_{n=1}^{\infty}\left(\left(N:_{R} M\right)^{-n}\right)^{-1} \\
& =\bigcap_{n=1}^{\infty}\left(N:_{R} M\right)_{\nu}^{n}=\bigcap_{n=1}^{\infty} N_{\nu}^{n} .
\end{aligned}
$$

Proposition 2.3. Let $R$ be an integral domain, $M$ a faithful multiplication $R$ module and $P$ a maximal submodule of $M$. Then $P^{n}$ is divisorial for each positive 
integer $n$ if and only if $\left(M:_{R_{T}} T(P)\right)=P_{0}$, where $P_{0}=\bigcap_{n=1}^{\infty} P^{n}$.

Proof. Let $P^{n}$ be a divisorial submodule of $M$ for each positive integer $n$. By Lemma $2.2, \bigcap_{n=1}^{\infty} P^{n}=\bigcap_{n=1}^{\infty}\left(P^{n}\right)_{\nu}=\left(M:_{R_{T}} T(P)\right)$.

For converse, assume that $P$ is a maximal submodule of $M$. If $P^{-1}=R$, then by [2, Lemma 1], $\left(P:_{R} M\right)^{-1}=R$. Hence by induction we have

$$
\begin{aligned}
P^{-n} & =\left(P:_{R} M\right)^{-n}=\left(R:_{T(R)}\left(P:_{R} M\right)^{n}\right) \\
& =\left(R:_{T(R)}\left(P:_{R} M\right)^{n-1}:_{R}\left(P:_{R} M\right)\right) \\
& =\left(\left(P:_{R} M\right)^{-(n-1)}:_{R}\left(P:_{R} M\right)\right) \\
& =\left(R:_{T(R)}\left(P:_{R} M\right)\right)=\left(P:_{R} M\right)^{-1}=P^{-1}=R .
\end{aligned}
$$

Thus

$$
\begin{aligned}
T(P) & =\bigcup_{n=1}^{\infty}\left(M:_{R_{T}}\left(P:_{R} M\right)^{n} M\right)=\bigcup_{n=1}^{\infty}\left(R:_{T(R)}\left(P:_{R} M\right)^{n}\right) \\
& =\bigcup_{n=1}^{\infty}\left(P:_{R} M\right)^{-n}=\bigcup_{n=1}^{\infty}\left(\left(P:_{R} M\right)^{-1}\right)^{n}=\bigcup_{n=1}^{\infty}\left(P^{-1}\right)^{n}=R .
\end{aligned}
$$

Then $\left(M:_{R_{T}} T(P)\right)=\left(M:_{R_{T}} R\right)=M$, which is a contradiction. Hence $P$ is a invertible submodule of $M$, [2, Proposition 13]. So by Lemma 2.1, $P$ is a finitely generated submodule of $M$. Then, by Lemma $2.1, P^{n}$ is a divisorial submodule of $M$ for each positive integer $n$.

Lemma 2.4. Let $R$ be an integral domain, $M$ a Prüfer faithful multiplication $R$ module and $P$ a maximal submodule of $M$. Then each power of $P$ is a divisorial submodule of $M$ if and only if $P^{2}$ is a divisorial submodule of $M$.

Proof. Assume that $P^{2}$ is a divisorial submodule of $M$. If $P$ is not invertible, then $P^{-1}=R,\left[2\right.$, Proposition 13]. Then, by [2, Lemma 1], $\left(P:_{R} M\right)^{-1}=R$. So, by [2, Lemma 1], we have

$$
\begin{aligned}
P^{-2} & =\left(P:_{R} M\right)^{-2}=\left(R:_{T(R)}\left(P:_{R} M\right)^{2}\right) \\
& =\left(R:_{T(R)}\left(P:_{R} M\right):_{R}\left(P:_{R} M\right)\right) \\
& =\left(\left(P:_{R} M\right)^{-1}:_{R}\left(P:_{R} M\right)\right) \\
& =\left(R:_{T(R)}\left(P:_{R} M\right)\right)=\left(P:_{R} M\right)^{-1}=R .
\end{aligned}
$$

Thus $P^{2}$ is not a divisorial submodule of $M$, which is a contradiction. Therefore $P$ is an invertible submodule of $M$ and so $P$ is a finitely generated submodule of $M$. Thus by Lemma 2.1, $P$ is a divisorial submodule of $M$ and so $P^{n}$ is a divisorial submodule of $M$ for each $n \geq 1$. The converse is clear.

It is shown that, if $M$ is a finitely generated multiplication $R$-module and $N$ a submodule of $M$, then $N$ is an idempotent submodule of $M$ if and only if $\left(N:_{R} M\right)$ is an idempotent ideal of $R$. 
Theorem 2.5. Let $R$ be an integral domain, $M$ a Prüfer faithful multiplication $R$-module and $P$ a maximal submodule of $M$. The following are equivalent:

(1) $P^{n}$ is a divisorial submodule of $M$ for each $n \geq 1$;

(2) $(M: T(P))=P_{0}$, where $P_{0}=\bigcap_{n=1}^{\infty} P^{n}$;

(3) $P^{2}$ is a divisorial submodule of $M$;

(4) Either $P^{-1} \subset T(P)$ or $P$ is a divisorial idempotent submodule of $M$.

Proof. (1) $\Leftrightarrow$ (2) Lemma 2.3.

(1) $\Leftrightarrow(3)$ Lemma 2.4.

$(1) \Rightarrow(4)$ Let $P^{-1}=T(P)$. Then, by [2, Lemma 1], $\left(P:_{R} M\right)^{-1}=P^{-1}=T(P)=$ $T\left(\left(P:_{R} N\right)\right)$. Thus, by [11, Theorem 4.1.19], $\left(P:_{R} M\right)$ is an idempotent ideal of $R$. Hence $P$ is an idempotent submodule of $M$.

$(4) \Rightarrow(1)$ Let $P^{-1} \subset T(P)$. If $P^{-1}=R$, then by [2, Lemma 1], $\left(P:_{R} M\right)^{-1}=R$. Hence

$$
\begin{aligned}
T(P) & =\bigcup_{n=1}^{\infty}\left(M:_{R_{T}}\left(P:_{R} M\right)^{n} M\right)=\bigcup_{n=1}^{\infty}\left(R:_{T(R)}\left(P:_{R} M\right)^{n}\right) \\
& =\bigcup_{n=1}^{\infty}\left(P:_{R} M\right)^{-n}=R
\end{aligned}
$$

which is a contradiction, because $R \subseteq P^{-1} \subset T(P)$. Therefore $P$ is an invertible submodule of $M$, [2, Proposition 13]. Then, by Lemma $2.1, P$ is a finitely generated submodule of $M$ and so $P$ is a divisorial submodule of $M$. Therefore, by Lemma 2.1, $P^{n}$ is divisorial submodule of $M$ for each $n \geq 1$.

Lemma 2.6. Let $R$ be an integral domain, $M$ a faithful multiplication $R$-module and $N$ a submodule of $M$. Then $N^{-1}=\left(N:_{T(R)} N\right)$ if and only if $N N^{-1}=N$.

Proof. Assume that $N^{-1}=\left(N:_{T(R)} N\right)$. Then $\left(N:_{R} M\right)^{-1}=\left(\left(N:_{R} M\right) M:_{T(R)}\right.$ $\left.\left(\begin{array}{ll}N:_{R} & M\end{array}\right) M\right)$ and so $\left(N:_{R} M\right)^{-1}\left(N:_{R} M\right) M=\left(\left(\begin{array}{lll}N & :_{R} & M\end{array}\right) M:_{T(R)}\left(N:_{R}\right.\right.$

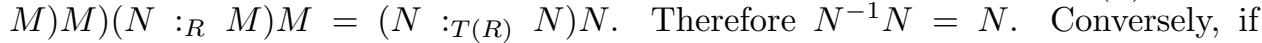
$N N^{-1}=N$, then by [2, Theorem 15], $\operatorname{Tr}_{M}(N)=N N^{-1}=N$. Therefore, by $[2$, Theorem 15], $N^{-1}=\left(N:_{T(R)} N\right)$.

Recall that a ring $R$ satisfies the radical trace property if $\operatorname{Tr}(I)=\sqrt{I I^{-1}}$ or $\operatorname{Tr}(I)=R$. It is shown that $R$ satisfies the radical trace property if and only if $R_{S}$ satisfies the radical trace property for each multiplicatively closed subset $S$ of $R$ ([11, Theorem 4.2.13]).

Definition 2.7. We say that an $R$-module $M$ satisfies radical trace property $(R T P)$ provided that $\operatorname{Tr}_{M}(N)=M$ or $\operatorname{Tr}_{M}(N)=\sqrt{N N^{-1}}$ for each submodule $N$ of $M$.

Theorem 2.8. Let $R$ be an integral domain and $M$ a faithful multiplication $R$ module. Then $M$ satisfy $R T P$ if and only if $R$ satisfies $R T P$.

Proof. Let $M$ satisfy $R T P$ and $I$ an ideal of $R$. Then $I M$ is a submodule of $M$. Therefore $(I M)(I M)^{-1}=M$ or $(I M)(I M)^{-1}=\sqrt{(I M)(I M)^{-1}}$. Hence, by 
[6, Lemma 6], $I I^{-1}=R$ or $I I^{-1}=\sqrt{I I^{-1}}$ and so $R$ satisfies $R T P$. Conversely, assume that $R$ satisfies $R T P$ and let $N$ be a submodule of $M$. Then $\left(N:_{R} M\right)$ is an ideal of $R$. Thus $\left(N:_{R} M\right)\left(N:_{R} M\right)^{-1}=R$ or $\left(N:_{R} M\right)\left(N:_{R} M\right)^{-1}=$ $\sqrt{\left(N:_{R} M\right)\left(N:_{R} M\right)^{-1}}$. So, by [6, Lemma 6$],\left(N:_{R} M\right)\left(N:_{R} M\right)^{-1} M=M$ or $\left(N:_{R} M\right)\left(N:_{R} M\right)^{-1} M=\sqrt{\left(N:_{R} M\right)\left(N:_{R} M\right)^{-1}} M$. Thus, by [2, Lemma 1], $N N^{-1}=M$ or $N N^{-1}=\sqrt{N N^{-1}}$. Therefore $M$ satisfies $R T P$.

Corollary 2.9. Let $R$ be an integral domain, $M$ a faithful multiplication $R$-module and $S$ a multiplicatively closed subset of $R$. Then $M_{S}$ satisfies RTP if and only if $R_{S}$ satisfies $R T P$.

Theorem 2.10. Let $M$ be a faithful multiplication $R$-module. Then the following are equivalent:

(1) $M$ satisfies RTP;

(2) For each multiplicatively closed subset $S$ of $R, M_{S}$ satisfies $R T P$;

(3) For each prime submodule $P$ of $M, \frac{M}{P}$ satisfies $R T P$.

Proof. (1) $\Rightarrow(2)$ Assume that $M$ satisfies $R T P$. Then, by Theorem $2.8, R$ satisfies $R T P$. So, by [11, Theorem 4.2.13], $R_{S}$ satisfies $R T P$. Therefore, by Corollary 2.9, $M_{S}$ satisfies $R T P$.

$(2) \Rightarrow(1)$ Set $S=\{1\}$, then $M=M_{S}$.

$(1) \Rightarrow(3)$ If $P$ is a maximal submodule of $M$, then $\frac{M}{P}$ is a simple module. Therefore assume that $P$ is a nonmaximal prime submodule of $M$. Let $N$ be a submodule of $M$ containing $P$ and $K^{\prime}:=\left(\frac{N}{P}\right)\left(\frac{N}{P}\right)^{-1} \neq \frac{M}{P}$. We show that $K^{\prime}$ is a radical submodule of $\frac{M}{P}$. Write $K^{\prime}=\frac{K}{P}$ for some submodule $K$ of $M$, where $K$ contains $P$. By [2, Proposition 15], we have

$$
\begin{aligned}
\frac{K^{-1}}{P} & =\left(\frac{M}{P}:_{R_{T}} \frac{K}{P}\right)=\left(\frac{M}{P}:_{R_{T}} K^{\prime}\right)=\left(K^{\prime}\right)^{-1}=\operatorname{Tr}_{\frac{M}{P}}\left(\frac{N}{P}\right)^{-1} \\
& =\left(\operatorname{Tr}_{\frac{M}{P}}\left(\frac{N}{P}\right):_{T(R)} \operatorname{Tr}_{\frac{M}{P}}\left(\frac{N}{P}\right)\right)=\left(K^{\prime}:_{T(R)} K^{\prime}\right) .
\end{aligned}
$$

Let $u \in K^{-1}$, then $(u+P) \frac{K}{P} \subseteq \frac{K}{P}$ and so $u K \subseteq K$. Thus $K^{-1} \subseteq\left(K:_{T(R)} K\right)$. On the other hand, by [2, Lemma 1$],\left(K:_{T(R)} K\right) \subseteq K^{-1}$. Hence $K^{-1}=\left(K:_{T(R)} K\right)$ and so by Lemma 2.6, $K K^{-1}=K(\neq M)$. Since $M$ satisfies $R T P$, then $K$ is a radical submodule of $M$. Consequently $\sqrt{K^{\prime}}=\sqrt{\frac{K}{P}}=\frac{\sqrt{K}}{P}=\frac{K}{P}=K^{\prime}$.

$(3) \Rightarrow(1)$ Set $P=0$, then $M \simeq \frac{M}{P}$.

It is obvious that if $I$ and $J$ are ideals of $R$, then $\left(I:_{R} J\right) M \subseteq\left(I M:_{T(R)} J M\right)$.

Lemma 2.11. Let $R$ be an integral domain, $M$ a faithful multiplication $R$-module and $Q$ a $P$-primary submodule of $M$. If $N$ is a submodule of $M$ containing $Q$ which is not contained in $P$, then $N^{-1} \subseteq\left(Q:_{T(R)} Q\right)$.

Proof. Since $Q$ is a $P$-primary submodule of $M$, then by [2, Lemma 4], $\left(Q:_{R} M\right)$ is a $\left(P:_{R} M\right)$-primary ideal of $R$. Therefore, by [11, Lemma 4.2.14], $\left(N:_{R} M\right)^{-1} \subseteq$ 
$\left(\left(Q:_{R} M\right):_{T(R)}\left(Q:_{R} M\right)\right)$. Since, by [2, Lemma 1], $N^{-1}=\left(N:_{R} M\right)^{-1}$, so $N^{-1} \subseteq\left(\left(Q:_{R} M\right):_{T(R)}\left(Q:_{R} M\right)\right) M \subseteq\left(Q:_{T(R)} Q\right)$.

Lemma 2.12. Let $R$ be an integral domain, $M$ a faithful multiplication $R$-module and $Q$ a $P$-primary submodule of $M$. Let $N$ is a submodule of $M$ such that $Q \subseteq N \subseteq$ $Q Q^{-1}$ and $N \nsubseteq P$, Then $N^{-1}=\left(Q Q^{-1}\right)^{-1}=\left(Q Q^{-1}:_{T(R)} Q Q^{-1}\right)=\left(Q:_{T(R)} Q\right)$. Proof. Since $Q \subseteq N \subseteq Q Q^{-1}$, then, by [2, Proposition 15] and Lemma 2.11, we have

$$
\left(Q:_{T(R)} Q\right) \subseteq\left(Q Q^{-1}:_{T(R)} Q Q^{-1}\right)=\left(Q Q^{-1}\right)^{-1} \subseteq N^{-1} \subseteq\left(Q:_{T(R)} Q\right)
$$

Therefore $N^{-1}=\left(Q Q^{-1}\right)^{-1}=\left(Q Q^{-1}:_{T(R)} Q Q^{-1}\right)=\left(Q:_{T(R)} Q\right)$.

Lemma 2.13. ([7, Lemma 3.6]) Let $R$ be an integral domain, $M$ a faithful multiplication $R$-module and $N, L$ submodules of $M$. Then $\left(N:_{R} M\right)\left(L:_{R} M\right)=\left(N L:_{R} M\right)$.

Proposition 2.14. Let $R$ be an integral domain and $M$ a faithful multiplication $R$-module. Then $M$ is a discrete Valuation module if and only if each P-primary submodule of $M$ is a power of $P$.

Proof. Assume that $M$ be a discrete Valuation module and $Q$ a $P$-primary submodule of $M$. Then, by [2, Lemma 4], $\left(Q:_{R} M\right)$ is $\left(P:_{R} M\right)$-primary ideal of $R$. So, By [12, Theorem 1], $R$ is a discrete valuation domain. Thus, by [11, Lemma 5.3.1] and Lemma 2.13, there exists a positive integer $n$ such that $\left(Q:_{R} M\right)=\left(P:_{R} M\right)^{n}=\left(P^{n}:_{R} M\right)$. Therefore $Q=P^{n}$.

Conversely, suppose that $Q$ be a $P$-primary submodule of $M$ such that for some positive integer $n, Q=P^{n}$. So, by [2, Lemma 4], $\left(Q:_{R} M\right)$ is $\left(P:_{R} M\right)$-primary ideal of $R$. If $M$ is not discrete Valuation module, then by [4, Theorem 1], $R$ is not a discrete Valuation domain. Thus, by [11, Lemma 5.3.1] and Lemma 2.13, $\left(Q:_{R} M\right) \neq\left(P:_{R} M\right)^{n}=\left(P^{n}:_{R} M\right)$ for each positive integer $n$. Therefore $Q \neq P^{n}$ for each positive integer $n$, which is a contradiction. $17.3]$

Compare the next results with [12, Theorem 7.2,Theorem 17.1 and Theorem

Lemma 2.15. Let $R$ ba an integral domain and $M$ a faithful multiplication $R$ module. Let $N$ and $K$ be two submodules of $M$ such that $N$ is invertible and $K \subseteq N$. Then there exists an ideal $I$ of $R$ such that $K=I N$.

Proof. Let $N$ and $K$ be two submodules of $M$ such that $N$ is invertible and $K \subseteq N$. Then $\left(N:_{R} M\right)$ and $\left(K:_{R} M\right)$ are ideals of $R$ and $\left(N:_{R} M\right)$ is an invertible ideal of $R$ and $\left(K:_{R} M\right) \subseteq\left(N:_{R} M\right)$. Thus, by [12, Theorem 7.2], there exists an ideal $I$ of $R$ such that $\left(K:_{R} M\right)=I\left(N:_{R} M\right)$. Therefore $K=\left(K:_{R} M\right) M=I\left(N:_{R}\right.$ $M) M=I N$.

Lemma 2.16. Let $R$ be an integral domain, $K$ a quotient field of $R$ such that $R \neq K, M$ a Valuation faithful multiplication $R$-module and $N$ a proper submodule of $M$. Then $P=\bigcap_{n=1}^{\infty} N^{n}$ is a prime submodule of $M$. 
Proof. It is obvious that $P=\bigcap_{n=1}^{\infty} N^{n}$ is a submodule of $M$. Since $N \neq M$, then $[N: M]$ is a proper ideal of $R$. Since $M$ is Valuation module, then $R$ is Valuation domain and so by $\left[12\right.$, Theorem 17.1] and Lemma 2.13, $P_{0}=\bigcap_{n=1}^{\infty}[N$ : $M]^{n}=\bigcap_{n=1}^{\infty}\left[N^{n}: M\right]$ is a prime ideal of $R$. Therefore, by [9, Lemma 2.10], $P=\bigcap_{n=1}^{\infty}\left[N^{n}: M\right] M=\bigcap_{n=1}^{\infty} N^{n}$ is a prime submodule of $M$.

Lemma 2.17. Let $R$ be an integral domain, $K$ a quotient field of $R$ such that $R \neq K$ and $M$ a Valuation faithful multiplication $R$-module. Then the product of two $P$-primary submodules of $M$ is a P-primary submodule of $M$.

Proof. Let $N_{1}$ and $N_{2}$ be two $P$-primary submodules of $M$. Clearly $N_{1} N_{2}$ is a submodule of $M$. Then, by [2, Lemma 1], $\left(N_{1}:_{R} M\right)$ and $\left(N_{2}:_{R} M\right)$ are $\left(P:_{R} M\right)$ primary ideals of $R$. Since $M$ is Valuation module, then $R$ is Valuation domain and so by [12, Theorem 17.3] and Lemma 2.13, $\left(N_{1}:_{R} M\right)\left(N_{2}:_{R} M\right)=\left(N_{1} N_{2}:_{R} M\right)$ is a $\left(P:_{R} M\right)$-primary ideal of $R$. Therefore by [2, Lemma 4], $N_{1} N_{2}=\left(N_{1} N_{2}: R\right.$ $M) M$ is a $P=\left(P:_{R} M\right) M$-primary submodule of $M$.

Theorem 2.18. Let $R$ be an integral domain, $K$ a quotient field of $R$ such that $R \neq K$ and $M$ a Valuation faithful multiplication $R$-module. If $P \neq P^{2}$ is a prime submodule of $M$, then each $P$-primary submodule of $M$ is a power of $P$.

Proof. Since $P$ is a prime submodule of $M$, then $P$ is a primary submodule of $M$. Also $\left(P:_{R} M\right)$ is a prime ideal of $R$, so $\left(P:_{R} M\right)$ is $\left(P:_{R} M\right)$-primary ideal of $R$. Then, by [2, Lemma 4], $P$ is $P$-primary submodule of $M$. So, by Lemma 2.17 , each power of $P$ is a $P$-primary submodule of $M$.

Now, let $Q$ be a $P$-primary submodule of $M$. Then by [2, Lemma 4], $\left(Q:_{R} M\right)$ is a $\left(P:_{R} M\right)$-primary ideal of $R$. If $Q \subseteq P^{n}$ for each positive integer $n$, then $Q \subseteq \bigcap_{n=1}^{\infty} P^{n}$ and by Lemma 2.16, $P_{0}=\bigcap_{n=1}^{\infty} P^{n}$ is a prime submodule of $M$. Since $P_{0} \subseteq P^{2} \neq P$, then $P_{0} \varsubsetneqq P$. Since $Q \subseteq \bigcap_{n=1}^{\infty} P^{n}=P_{0}$, then $\left(Q:_{R} M\right) \subseteq$ $\left(P_{0}:_{R} M\right)$ and so $\sqrt{\left(Q:_{R} M\right)} \subseteq \sqrt{\left(P_{0}:_{R} M\right)}$. Thus $\left(P:_{R} M\right) \subseteq\left(P 0:_{R} M\right)$ and so $P=\left(P:_{R} M\right) M \subseteq\left(P_{0}:_{R} M\right) M=P_{0}$, which is a contradiction. Therefore there exists a positive integer $n$ such that $Q \nsubseteq P^{n}$. Since $M$ is a Valuation module, then $P^{n} \subseteq Q$. Suppose that $m$ is the smallest positive integer such that $P^{m} \subseteq Q$. Thus $Q \varsubsetneqq P^{m-1}$. choose $x \in M$ such that $x \in P^{m-1}$ and $x \notin Q$. Since $M$ is a Valuation module, then $Q \subseteq(x)$. On the other hand $(x)$ is a principal submodule of $M$ and so is an invertible submodule of $M$. Therefore, by Lemma 2.15, there exists an ideal $I$ of $R$ such that $Q=I$.(x). Thus $Q \subseteq I$. So for each $a \in I$, ax $\in I .(x)=Q$. We know that $R a$ is an ideal of $R$ and $a \in R a \subseteq R$. Since $a x \in Q$ and $Q$ is a $P$-primary submodule of $M$ and $x \notin Q$, so $a \in \sqrt{\left(Q:_{R} M\right)}=P$. Thus $I \subseteq P$. Then $Q=I .(x) \subseteq P P^{m-1}=P^{m}$. Therefore $Q=P^{m}$.

\section{When $(N \cap L)^{-1}$ is a Ring?}

Theorem 3.1. Let $R$ be an integral domain, $M$ a faithful multiplication $R$-module and $N, L$ coprime radical submodules of $M$. Then the following are equivalent:

(1) $N^{-1}$ and $L^{-1}$ are rings. 
(2) $(N \cap L)^{-1}$ and $(N+L)^{-1}$ are rings.

Moreover, $(N+L)^{-1}=\left(N+L:_{T(R)} N+L\right)$.

Proof. (1) $\Rightarrow(2)$ Let $N^{-1}$ and $L^{-1}$ be rings. Then, by [2, Lemma 1], $\left(N:_{R} M\right)^{-1}$ and $\left(L:_{R} M\right)^{-1}$ are rings. So $\left(N:_{R} M\right)^{-1}+\left(L:_{R} M\right)^{-1}$ is a ring. Thus $N^{-1}+L^{-1}$ is a ring. Since $N+L=M$, therefore by [2, Lemma 2], $(N \cap L)^{-1}=N^{-1}+L^{-1}$ is a ring. Also, by [2, Lemma 2] we have

$$
\begin{aligned}
(N+L)^{-1} & =\left(\left(N:_{R} M\right) M+\left(L:_{R} M\right) M\right)^{-1}=\left(\left(\left(N:_{R} M\right)+\left(L:_{R} M\right)\right) M\right)^{-1} \\
& =\left(\left(N:_{R} M\right)+\left(L:_{R} M\right)\right)^{-1}=\left(N:_{R} M\right)^{-1} \cap\left(L:_{R} M\right)^{-1} \\
& =N^{-1} \cap L^{-1} .
\end{aligned}
$$

Since $N^{-1} \cap L^{-1}$ is a ring, then $(N+L)^{-1}$ is a ring.

$(2) \Rightarrow(1)$ Let $(N \cap L)^{-1}$ and $(N+L)^{-1}$ are rings. Then, by [2, Lemma 1], $\left(N \cap L:_{R}\right.$ $M)^{-1}$ and $\left(N+L:_{R} M\right)^{-1}$ are rings. Therefore, by [10, Theorem 3.4], $\left(N:_{R} M\right)^{-1}$ and $\left(L:_{R} M\right)^{-1}$ are rings. So, by [2, Lemma 1$], N^{-1}$ and $L^{-1}$ are rings.

Now we show that $(N+L)^{-1}=\left(N+L:_{T(R)} N+L\right)$. By [2, Lemma 1], $(N+$ $\left.L:_{T(R)} N+L\right) \subseteq(N+L)^{-1}$. For the other inclusion, by [2, Lemma 2], we have $(N+L)^{-1}=N^{-1} \cap L^{-1}$. Let $x \in N^{-1} \cap L^{-1}$, then by [2, Proposition 11] $x \in N^{-1}=\left(N:_{T(R)} N\right)$ and so $x N \subseteq N$. Similary $x \in L^{-1}=\left(L:_{T(R)} L\right)$, so $x L \subseteq L$ and thus $x(N+L)=x N+x L \subseteq N+L$ and therefore $x \in\left(N+L:_{T(R)}\right.$ $N+L)$.

By induction we have the following corollary.

Corollary 3.2. Let $R$ be an integral domain and $M$ a faithful multiplication $R$ module. Let $N_{1}, \ldots, N_{n}$ be radical submodules of $M$ such that $N_{i}+N_{j}=M$ for $1 \leq i, j \leq n$ and $i \neq j$. If $N_{1}^{-1}, \ldots, N_{n}^{-1}$ are rings, then $\left(N_{1} \cap \ldots \cap N_{n}\right)^{-1}$ is a ring.

Proposition 3.3. Let $R$ be an integral domain, $M$ a faithful multiplication $R$ module and $N$ a nonzero submodule of $M$ such that $N^{-1}$ is a ring. Then $(\sqrt{N})^{-1}$ is a ring and $(\sqrt{N})^{-1}=\left(\sqrt{N}:_{T(R)} \sqrt{N}\right)$.

Proof. Suppose that $x \in(\sqrt{N})^{-1}$. For each $a \in \sqrt{N}=\sqrt{\left(N:_{R} M\right)} M$ there exists a positive integer number $n$ such that $a^{n} \in\left(N:_{R} M\right) M=N$. Since $N=\left(N:_{R}\right.$ $M) M \subseteq \sqrt{\left(N:_{R} M\right)} M=\sqrt{N}$, then $(\sqrt{N})^{-1} \subseteq N^{-1}$ and so $x \in N^{-1}$. Since $N^{-1}$ is a ring, then $x^{2 n} \in N^{-1}$. So $a^{n} x^{2 n} \in N N^{-1} \subseteq M$. Thus $(a x)^{2 n}=a^{n}\left(a^{n} x^{2 n}\right) \in$ $N M \subseteq N$ and it follows that $a x \in \sqrt{N}$. Then $x \sqrt{N} \subseteq \sqrt{N}$ and so $x \in(\sqrt{N}: T(R)$ $\sqrt{N})$. On the other hand, by [2, Lemma 1], $\left(\sqrt{N}:_{T(R)} \sqrt{N}\right) \subseteq(\sqrt{N})^{-1}$. Therefore $(\sqrt{N})^{-1}=\left(\sqrt{N}:_{T(R)} \sqrt{N}\right)$ is a ring.

Corollary 3.4. Let $R$ be a integral domain, $M$ a faithful multiplication $R$-module and $N, L$ coprime submodules of $M$. If $N^{-1}$ and $L^{-1}$ are rings, then $(\sqrt{N} \cap \sqrt{L})^{-1}$ and $(\sqrt{N}+\sqrt{L})^{-1}$ are rings.

Proof. Let $N^{-1}$ and $L^{-1}$ be rings. Then, by Proposition 3.3, $(\sqrt{N})^{-1}$ and $(\sqrt{L})^{-1}$ are rings. Since $M=N+L \subseteq \sqrt{N}+\sqrt{L} \subseteq M$, then $\sqrt{N}+\sqrt{L}=M$. Moreover $\sqrt{N}$ and $\sqrt{L}$ are radical submodules. Therefore, by Theorem 3.1, we are done. 
Lemma 3.5. Let $R$ be a ring and $M$ an R-module. Then $\left(N_{\nu}\right)^{-1}=N^{-1}$.

Proof. Since $N N^{-1} \subseteq M$, then $N \subseteq\left(M:_{R_{T}} N^{-1}\right)=\left(N^{-1}\right)^{-1}=N_{\nu}$. So $\left(N_{\nu}\right)^{-1} \subseteq$ $N^{-1}$. For the other inclusion, let $x \in N_{\nu}=\left(M:_{R_{T}} N^{-1}\right)$. Then $x N^{-1} \subseteq M$ and hence $N_{\nu} N^{-1} \subseteq M$. Thus $N^{-1} \subseteq\left(M:_{R_{T}} N_{\nu}\right)=\left(N_{\nu}\right)^{-1}$. Therefore $\left(N_{\nu}\right)^{-1}=$ $N^{-1}$.

Theorem 3.6. Let $R$ be an integral domain, $M$ a faithful multiplication $R$-module and $N, K$ coprime submodules of $M$ such that $N^{-1} \cap K^{-1}=R$. Then the following are equivalent:

(1) $N^{-1}$ and $L^{-1}$ are rings.

(2) $(N \cap L)^{-1}$ is a ring.

(3) $\left(N_{\nu} \cap L_{\nu}\right)^{-1}$ is a ring.

Moreover, $(N \cap L)^{-1}=\left(N_{\nu} \cap L_{\nu}\right)^{-1}=(N L)^{-1}=\left(N_{\nu} L_{\nu}\right)^{-1}$.

Proof. (1) $\Rightarrow(2)$ Let $N^{-1}$ and $L^{-1}$ be rings. Then $N^{-1}+L^{-1}$ is a ring. Therefore, by [2, Lemma 2], $(N \cap L)^{-1}=N^{-1}+L^{-1}$ is a ring.

(2) $\Rightarrow(1)$ Let $(N \cap L)^{-1}$ be a ring. Then, by [2, Lemma 1], $\left(N \cap L:_{R} M\right)^{-1}=$ $\left(\left(N:_{R} M\right) \cap\left(L:_{R} M\right)\right)^{-1}$ is a ring. Hence, by [10, Theorem 3.7], $\left(N:_{R} M\right)^{-1}$ and $\left(L:_{R} M\right)^{-1}$ are rings. Therefore, by [2, Lemma 1], $N^{-1}$ and $L^{-1}$ are rings.

If $N^{-1}$ and $L^{-1}$ are rings, then, by Lemma $3.5,\left(N_{\nu}\right)^{-1}$ and $\left(L_{\nu}\right)^{-1}$ are rings. Since (1) and (2) are equivalent, it follows that $\left(N_{\nu} \cap L_{\nu}\right)^{-1}$ is a ring.

For the last equality, by [2, Lemma 1] we have $N^{-1}=\left(N:_{R} M\right)^{-1}$ and $N_{\nu}=\left(N:_{R}\right.$ $M)_{\nu}$. Therefore $(N \cap L)^{-1}=\left(N_{\nu} \cap L_{\nu}\right)^{-1}=(N L)^{-1}=\left(N_{\nu} L_{\nu}\right)^{-1}$.

Proposition 3.7. Let $R$ be an integral domain, $M$ a faithful multiplication $R$ module and $N$ a radical submodule of $M$ such that $N=K \cap L$ for submodules $K, L$ of $M$. Then $N^{-1}$ is a ring if and only if there are radical submodules $K_{1} \supseteq K$ and $L_{1} \supseteq L$ of $M$ such that $N=K_{1} \cap L_{1}$ and $K_{1}^{-1}$ and $L_{1}^{-1}$ are rings.

Proof. Let $N$ be a radical submodule of $M$ such that $N=K \cap L$ for submodules $K, L$ of $M$. Then $\left(N:_{R} M\right)$ is a radical ideal of $R$ and $\left(N:_{R} M\right)=\left(K \cap L:_{R} M\right)=$ $\left(K:_{R} M\right) \cap\left(L:_{R} M\right)$. Now, if $N^{-1}$ is a ring, then by [2, Lemma 1], $\left(N:_{R} M\right)^{-1}$ is a ring and so by [10, Corollary 3.12], there are radical ideals $A \supseteq\left(K:_{R} M\right)$ and $B \supseteq\left(L:_{R} M\right)$ such that $\left(N:_{R} M\right)=A \cap B$ and $A^{-1}$ and $B^{-1}$ are rings. Therefore, there exist radical submodules $A M \supseteq\left(K:_{R} M\right) M=K$ and $B M \supseteq\left(L:_{R} M\right) M=$ $L$ such that $N=A M \cap B M$ and $A^{-1}$ and $B^{-1}$ are rings, by [2, Lemma 1].

Conversely, suppose that there are radical submodules $K_{1} \supseteq K$ and $L_{1} \supseteq L$ of $M$ such that $N=K_{1} \cap L_{1}$ and $K_{1}^{-1}$ and $L_{1}^{-1}$ are rings. Then, by [2, Lemma 1] and [6, Lemma 6], there are radical ideals $\left(K_{1}:_{R} M\right) \supseteq\left(K:_{R} M\right)$ and $\left(L_{1}:_{R} M\right) \supseteq$ $\left(L:_{R} M\right)$ of $R$ such that $\left(N:_{R} M\right)=\left(K_{1}:_{R} M\right) \cap\left(L_{1}:_{R} M\right)$ and $\left(K_{1}:_{R} M\right)^{-1}$ and $\left(L_{1}:_{R} M\right)^{-1}$ are rings. Therefore, by [10, Corollary 3.12], $\left(N:_{R} M\right)^{-1}$ is a ring and so by [2, Lemma 1$], N^{-1}$ is a ring.

Definition 3.8. Let $R$ be an ring, $M$ an $R$-module and $\left\{K_{\alpha}\right\}_{\alpha \in \Lambda}$ a non-empty set of prime submodules of $M$. We say that $N=\bigcap_{\alpha \in \Lambda} K_{\alpha}$ is irredundant, if for each $\beta \in \Lambda, \bigcap_{\alpha \neq \beta} K_{\alpha} \nsubseteq K_{\beta}$. 
Lemma 3.9. Let $R$ be an integral domain, $M$ a faithful multiplication $R$-module and $\left\{K_{\alpha}\right\}_{\alpha \in \Lambda}$ a non-empty set of prime submodules of $M$. Then $N=\bigcap_{\alpha \in \Lambda} K_{\alpha}$ is an irredundant submodule of $M$ if and only if $\left(N:_{R} M\right)=\left(\bigcap_{\alpha \in \Lambda} K_{\alpha}:_{R} M\right)$ is an irredundant ideal of $R$.

Proof. Let $N=\bigcap_{\alpha \in \Lambda} K_{\alpha}$ be an irredundant submodule of $M$. Since $K_{\alpha}$ is a prime submodule of $M$, then $\left(K_{\alpha}:_{R} M\right)$ is a prime ideal of $R$. If there exists $\beta \in \Lambda$ such that $\left(\bigcap_{\alpha \in \Lambda} K_{\alpha}:_{R} M\right) \subseteq\left(K_{\beta}:_{R} M\right)$, then $\bigcap_{\alpha \neq \beta} K_{\alpha}=\left(\bigcap_{\alpha \neq \beta} K_{\alpha}:_{R} M\right) M \subseteq$ $\left(K_{\beta}:_{R} M\right) M=K_{\beta}$, which is a contradiction. Therefore, $\left(N:_{R} M\right)=\left(\bigcap_{\alpha \in \Lambda} K_{\alpha}:_{R}\right.$ $M)$ is an irredundant ideal of $R$. The converse is similar.

Theorem 3.10. Let $R$ be an integral domain, $M$ a faithful multiplication $R$-module and $\left\{K_{\alpha}\right\}_{\alpha \in \Lambda}$ a non-empty set of prime submodules of $M$. If $N=\bigcap_{\alpha \in \Lambda} K_{\alpha}$ is a nonzero and irredundant submodule of $M$, then the following are equivalent:

(1) $N^{-1}$ is a ring;

(2) For each $\alpha \in \Lambda, K_{\alpha}^{-1}$ is a ring;

(3) For each non-empty subset $\Gamma$ of $\Lambda,\left(\bigcap_{\alpha \in \Gamma} K_{\alpha}\right)^{-1}$ is a ring.

Proof. Let $\left\{K_{\alpha}\right\}_{\alpha \in \Lambda}$ be a non-empty set of prime submodules of $M$. Then $\left\{\left(\begin{array}{ll}K_{\alpha}::_{R} & M\end{array}\right)\right\}_{\alpha \in \Lambda}$ is a non-empty set of prime ideals of $R$. If $N=\bigcap_{\alpha \in \Lambda} K_{\alpha}$ is a nonzero and irredundant submodule of $M$, then by Lemma 3.9, $\left(N:_{R} M\right)=$ $\left(\bigcap_{\alpha \in \Lambda} K_{\alpha}:_{R} M\right)=\bigcap_{\alpha \in \Lambda}\left(K_{\alpha}:_{R} M\right)$ is a nonzero irredundant ideal of $R$.

$(1) \Rightarrow(2)$ Let $N^{-1}$ be a ring. Then, by [2, Lemma 1], $\left(N:_{R} M\right)^{-1}$ is a ring. Therefore, by [10, Proposition 3.13], $\left(K_{\alpha}:_{R} M\right)^{-1}$ is a ring and so by [2, Lemma $1], K_{\alpha}^{-1}$ is a ring.

(2) $\Rightarrow$ (3) Let $\Gamma$ be a non-empty subset of $\Lambda$ and $L=\bigcap_{\alpha \in \Gamma} K_{\alpha}$. Then $\left(L:_{R}\right.$ $M)=\left(\bigcap_{\alpha \in \Gamma} K_{\alpha}:_{R} M\right)=\bigcap_{\alpha \in \Gamma}\left(K_{\alpha}:_{R} M\right)$. Therefore, by [10, Proposition 3.13],

$\left(L:_{R} M\right)^{-1}$ is a ring and so by [2, Lemma 1$], L^{-1}$ is a ring.

$(3) \Rightarrow(2)$ It is obvious.

\section{References}

[1] M. M. Ali, Invertibility of multiplication modules, New Zealand J. Math., 35(2006), 17-29.

[2] M. M. Ali, Invertibility of multiplication modules $\Pi$, New Zealand J. Math., 39(2009), 45-64.

[3] M. M. Ali, Some remarks on generalized GCD domains, Comm. Algebra, 36(2008), 142-164.

[4] M. M. Ali, Invertibility of multiplication modules III, New Zealand J. Math., 39(2009), 139-213.

[5] M. M. Ali, Idempotent and nilpotent submodules of multiplication modules, Comm. Algebra, 36(2008), 4620-4642.

[6] M. M. Ali, The transform formula for submodules of multiplication modules, New Zealand J. Math., 41(2011), 25-37. 
[7] R. Ameri, On the prime submodules of multiplication modules, Internat. J. Math. Math. Sci., 27(2003), 1715-1724.

[8] A. Barnard, Multiplication modules, J. Algebra, 71(1981), 174-178.

[9] Z. A. El-Bast and P. F. Smith, Multiplication modules, Comm. Algebra, 16(1998), 755-799.

[10] E. Houston, S. Kabbaj, T. Lucas and A. Mimouni When is the dual of an ideal a ring?, J. Algebra, 225(2000), 429-450.

[11] M. Fontana, J. Hukaba and I. Papick, Prüfer Domains, Marcel Dekker, (1997).

[12] R. Gilmer, Multiplicative ideal theory, Marcel Dekker: New York, (1972).

[13] M. D. Larsen and P. J. MacCaarthy, Multiplication theory of ideal, Academic Press: New York, (1971).

[14] A. G. Naoum, Flat modules and multiolication modules, Periodica. Math. Hungar., 21(1990), 309-317.

[15] A. G. Naoum and F. H. Al-Alwan, Dedekind modules, Comm. Algebra, 24(1996), 225-230.

[16] P. F. Smith, Multiplication modules, Comm. Algebra, 16(1988), 755-799.

[17] P. F. Smith, Some remarks on multiplication modules, Arch. der. Math., 50(1988), 223-235. 